\title{
An analysis of transportation system mechanisms using the agent-based simulation
}

\author{
S. Nakayama \\ Department of Civil Engineering, Kanazawa University, Japan
}

\begin{abstract}
Transportation systems in general consist of many agents who choose their behaviors through learning based on their experiences and information provided. The agents interact mutually through the system, and the system must be dynamic and complex. The agent-based simulation is one of the methods for examining such a complex system. The simulation enables us to model the transportation system relatively flexibly. Assuming that agents reason and learn inductively based on their experiences, agent-based transportation system simulation models are developed. Each agent learns how best to choose a route based on his experiences, and the behavior of such agents and the mechanism of the transportation system are examined through simulation experiments.
\end{abstract}

Keywords: agent-based simulation, transportation system analysis, network equilibrium, rule-based reasoning, route choice.

\section{Introduction}

The behavior of a transportation system results from an aggregation of each individual's travel behavior. The agent of travel behavior cannot predict the traffic state exactly before the trip, but obtains ex post facto information such as how many minutes it costs after the trip. So, he learns how to choose a route, departure time, or other travel choice based on his experiences. This may include prediction of traffic state before the trip. The state of the transportation system is given by an aggregation of these travel behaviors. The agents interact mutually through the traffic state, recording travel times.

We cannot appreciate or understand the mechanism of the system that includes nonlinear interaction by analyzing parts or elements of the system separately [1]. We have to investigate the whole system simultaneously. This 
indicates that we cannot understand the transportation system even if we examine the learning mechanism or travel behavior separately. We have to treat agents learning, their (nonlinear) interaction and the system dynamics simultaneously. An ordinary traffic simulation without agents learning via their experiences is not necessarily enough to investigate the transportation system fully in the sense of day-to-day dynamics because the nonlinear interaction is not described.

An agent-based simulation is one of the useful and effective approaches to examine such a system. It is said that the agent is an intelligent entity and a system that senses its environment and acts upon it [2]. Some researchers including me applied the agent-based simulation to the area of transportation engineering [3-7]. In this paper, an if-then rule system is adopted as a decisionmaking agent. The agent devises how to decide his behavior through his experiences using if-then rules. This includes formation of decision-making processes as well as learning. The if-then rule system is updated based on the travel results as the agent learns. Thus, the agent is initially endowed only with a basic framework for decision-making, and forms his own decision-making process. We shall call such a learning agent an "adaptive agent." This is different from the others' previous studies [5-7].

This study is concerned with the case where only route choice is involved. The agent attempts to arrive at his destination as fast as possible and learning how to choose the route based on his experiences. The agent in this paper chooses a route based on the if-then rules. Learning is that the if-then rule system of the individual agent is updated. The simulation with the learning if-then rule system is developed and the findings and understandings of the transportation system with adaptive agents are provided.

\section{Agent-based simulation model}

In this paper, each agent travels daily from a fixed origin, $\mathrm{O}$, to a destination, $\mathrm{D}$, and the only decision element is route choice for simplicity. The model consists of an agent sub-model and a traffic state sub-model as Fig. 1 shows. The former simulates each agent's route choice and learning, while the latter determines traffic state based on agents' route choices and evaluates the travel time experienced by each agent.

\subsection{Agent sub-model}

Assume agents memorize the fastest routes for the latest $m$ days. In reality, drivers may remember much more information such as the experienced travel time. They only memorize the fastest routes for the latest $m$ days in this paper. The agent chooses a route based on his memories, that is, he considers the history of the fastest routes only.

The agent sub-model is almost the same as my previous model [4]. The difference between this model and the previous one is the information the agent obtains and considers. Therefore, the structure of the system and if-then rules are 
revised. The agent sub-model performs the following: 1) scan the agent's memory and identify the if-then rules that apply (or, "activate") to the fastest routes in the past $m$ days, and select the route which the rule indicates, 2) if there are more than one if-then rule that activates, select that rule which has the highest "superiority" value (described below) and choose the route indicated by that rule, 3) choose a route randomly if there is no rule that activates, and 4) update rules and their superiority values.

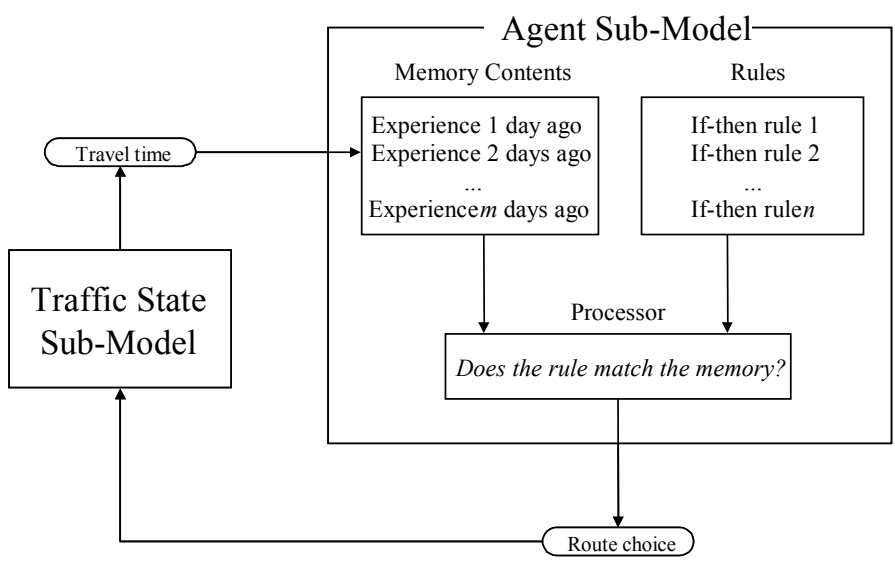

Figure 1: $\quad$ Outline of the simulation model.

Agents memorize the fastest routes for the latest $m$ days, and the memory can be coded as a set of bits, $x_{i}$ 's, where $x_{i}$ refers to the fastest route on the previous $i$ th day. An if-then rule consists of a condition and an action. The action part of an if-then rule contains the route, $y$, which the rule instructs the agent to take. The condition part comprises a set of bits, $x_{i}$ 's, where $x_{i}$ refers to the fastest route on the previous $i$ th day. This is the same structure as the memory described above. The condition implied by the $x_{i}$ 's is checked against the data in the memory, and a rule "activates" if the $m$ bits of the condition part which correspond to the $m$ pieces of memory from the last $m$ days. Fig. 2 illustrates an example of if-then rules in the case of 1 OD 2 route network (the network has 1 OD pair and the OD connects 2 routes) and $m=3$. In this case, two pieces of ifthen rules always activate.

Suppose that an agent have the memory that on the latest 3 days Route 1 was the fastest. In this case, the memory is coded as $\left[x_{1}, x_{2}, x_{3}\right]=[\mathrm{R} 1, \mathrm{R} 1, \mathrm{R} 1]$ and in Fig. 2, the condition parts of Rule 1 and Rule 1' are the same as the memory and these two rules are activated. If the superiority of Rule 1 is higher than Rule 1', the agent chooses the route Rule 1 instructs, that is, he chooses Route 1. Reversely, if the superiority of Rule 1' is higher than Rule 1, the agent chooses the route Rule 1' instructs.

How well each rule is performing is evaluated using the following superiority indicator. If there are more than one if-then rules that activate, it is logical to assume that an agent should apply the rule that has provided good instructions 


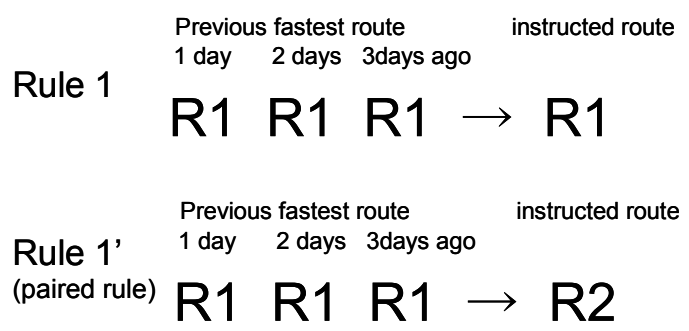

Figure 2: $\quad$ If-then rules with $m=3$.

more frequently in the past. The superiority indicator is used to judge which rule should be applied. The indicator is a weighted average of the travel times experienced on the route instructed by the rule, and is defined by applying the following recursive relationship each time the rule is used:

where

$$
f_{j}^{i+1}=c f_{j}^{i}+\delta_{j}^{i}
$$

$f_{j}^{i}=$ the superiority of the if-then rule $j$ on Day $i$

$\delta_{j}{ }^{i}=$ if the route that the if-then rule $j$ instructs is the fastest on Day $i, \delta_{j}^{i}$ takes $+a$; otherwise, $\delta_{j}^{i}$ takes $-a$.

$c=$ positive parameter $(0 \leq c \leq 1)$

$a=$ positive parameter $(a>0)$

The above equation means that the superiority increases if the route chosen is the fastest; otherwise, it decreases. The value of the parameter $a$ does not make a difference so much if it is positive. The larger is the superiority indicator, the better has the rule performed in the past. The parameter $c$ represents the rate at which the superiority indicator is updated; the larger is its value, the faster does the superiority indicate change its value. When superior indicators change their values rapidly, so do the rules themselves.

Note that if the rule was used and instructed the route the agent took on Day $i$, the rule is updated according to Eq. (1) prior to Day $i+1$ and the other rules are not.

\subsection{Traffic state sub-model}

Route choices made by the respective agents in the agent sub-model are aggregated and traffic volume is determined for each route. Travel time is then calculated for each route using the following formulation by the Bureau of Public Roads (BPR):

$$
t(x)=t_{f}\left\{1+\alpha(x / C)^{\beta}\right\}
$$

where $t$ is the travel time to traverse a network route, $x$ the traffic volume on the route, that is, the number of agents traveling on the route, $C$ the route capacity, $t_{f}$ the free-flow route travel time, and $\alpha$ and $\beta$ are constant parameters.

\section{Simulation results}

The model system described above was applied to the simple transportation network that has a pair of OD connected by two links (routes). Some simulation 
experiments were performed assuming that a total of 200 agents travel daily on the two-link network, making exactly one trip each day. Assume that all agents choose their routes independently without any knowledge of the other agents' choices. The number of days travel time information is stored in an agent's memory, $m$, is set to 3 , and the number of if-then rules an agent has is 16 (= $\left.2 * 2^{3}\right)$. At the initial setting, the value of parameter $c$ is given to each agent. The parameter, $c$, is uniformly distributed in the range from 0 to 1 , and is generally different among agents. After the value is provided, it is fixed during the simulation. The positive parameter, $a$, is set to 0.5 .

The example network has one OD pair and the OD pair connects by two links. The travel time functions of links are: $t_{1}(x)=20\left\{1+2(x / 200)^{2}\right\}$ and $t_{2}(x)=$ $10\left\{1+2(x / 100)^{2}\right\}$ where $t_{i}(x)$ denotes the travel time on the $i$-th link.

The network is simple and the number of agents is not so many. This simplified representation in the simulation analysis of this study is, however, considered to be sufficient as the objective of the study is to appreciate the behavior of the system. It is believed that making the model more detailed or realistic is not necessarily helpful in gaining insights into the mechanisms of complex systems. There would be cases where a model system which focuses on the most relevant factors while disregarding elements of lesser significance may better aid in gaining an understanding of the system behavior.

Wardrop's equilibrium [8] is the situation in which the travel times of both links are equal in this example setting. In the simulation, both travel times are 30.0 at equilibrium and traffic volumes are both 100. Fig. 3 shows the travel times of both links through Day 400. From the figure, we found that at the start of simulation the system oscillates ferociously because all agents have no knowledge of the system, but after Day 200, the system converges to Wardrop's equilibrium. This is because through learning, the agents try choosing faster link, and finally, the travel times of both links become equal. Fig. 4 shows the histogram on how many times the agents choose Link 1 until Day 400. While there are some agents who choose Link 1 almost every day, there are some other agents who continue to take Link 2. Thus, which link the agents choose is various among agents. This represents agents' heterogeneity.

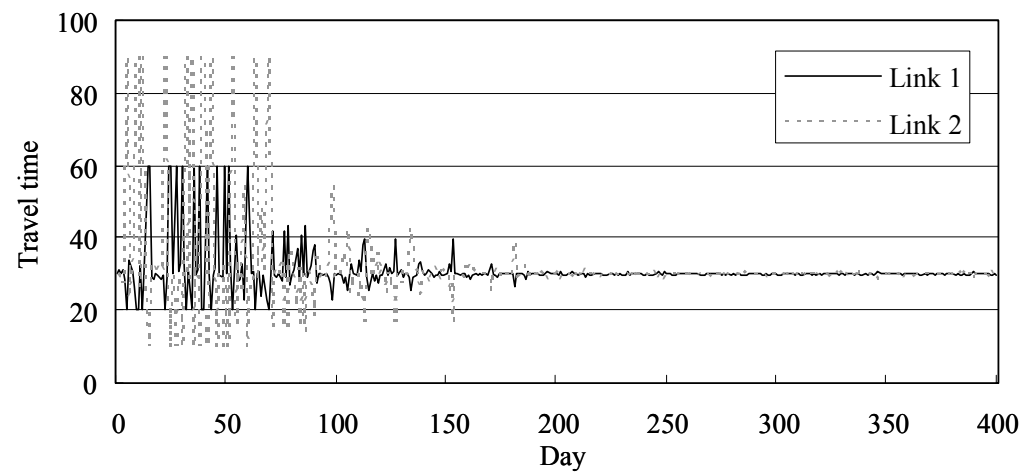

Figure 3: $\quad$ Travel times in the simulation. 


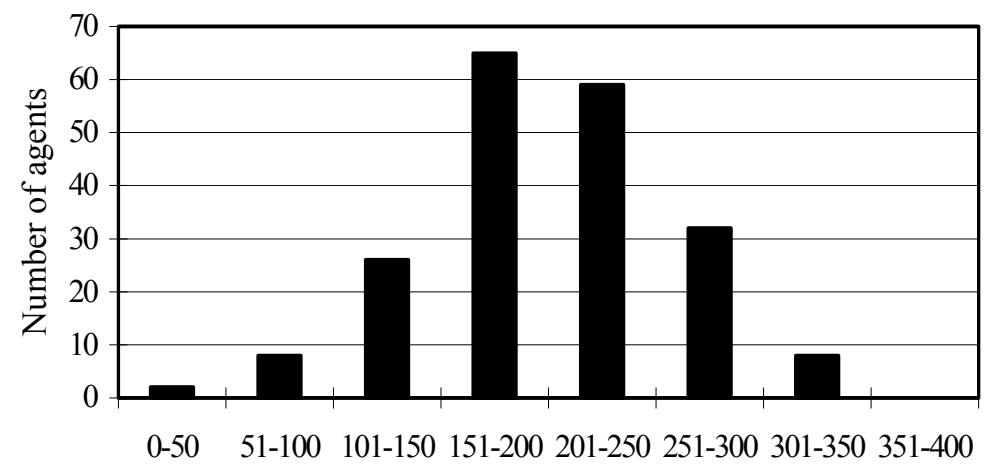

Figure 4: $\quad$ Frequency of choosing link 1 until day 400.

Most agents continue to use one of the two rules that have the same condition part. But, the used rules are also various among agents and each rule is evenly used. Heterogeneity of agents seems to contribute to reach Wardrop's equilibrium.

The agents try taking the fastest link. The "grade" of agent is how many times the agent chooses the fastest link (faster link in this two-link network). Fig. 5 shows the histogram on how many times the agents choose the fastest link until Day 400 . The average is 214.3 , the minimum is $181(45.0 \%)$, and the maximum is $240(60.3 \%)$. The standard deviation is 10.9 . The difference of the grades is not so small. In this simulation, all agents can store the information for 3 days, and the capability is the same among agents. Also, the information, that is, which link has been the fastest, is the same. The differences among agents are the parameter $c$ in Eq. (1) and the history of chosen links. It is found that there is no

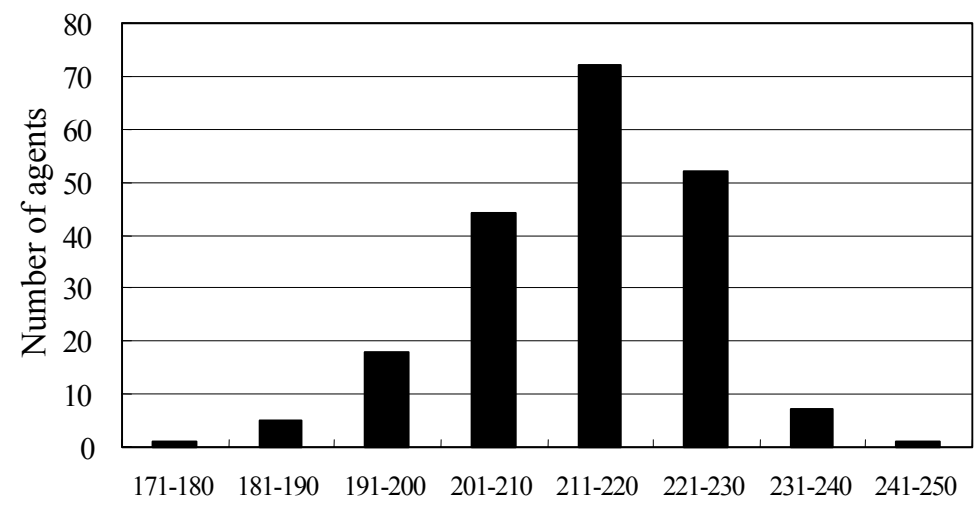

Figure 5: $\quad$ Frequency of choosing the fastest link (faster link) until day 400. 
correlation between them. We made another simulation where the value of the parameter $c$ is the same among agents. In this case, the difference of grades among agent is not small like Fig. 5, too. This result means that even if the agent has the same information processing ability and have the same information, not a small difference of grade or result is made, and implies that even though people use the route guidance system or navigation system with the same ability, some can take the fastest route many times while some other take the fastest route less times. In reality, we have different quantity and quality of information and have different ability of navigation systems. The grade may be much more various among travelers or commuters in the real network. Needless to say, these results come from the simple and ideal network case. We have to survey the network behavior and route choice behavior in the real world and examine these findings very carefully as future works.

\section{Conclusions}

Transportation system generally consists of many agents who learn based on their experiences and information provided. In this study, we developed an agent-based transportation system simulation model. In the model, the agent learns which route to choose based on his experiences. Then, we applied the simulation model to a simple transportation network, and examined the behavior of agents and network flow. The results of the simulation experiments can be summarized as follows: 1) the system converges to Wardrop equilibrium on average; 2) the grades (the number of times of choosing the fastest route) are various among the agents; 3 ) the difference of the grades occurs contingently.

As a future work, we will have to incorporate departure time choice and predicted travel time information to the simulation and examine time-to-time dynamics of the transportation system. Also, we have to apply the model system to more general cases, e.g. large-scale network and $m$ is various among agents.

\section{Acknowledgements}

I would like to express my gratitude to Prof. Ryuichi Kitamura (Kyoto University) and Prof. Jun-ichi Takayama (Kanazawa University) for providing valuable comments and advices. I am also grateful to Mr. Tatsuo Sato for his computational support.

\section{References}

[1] von Bertalanffy, L., General System Theory: Foundations, Development, Applications, Penguin Press: London, 1971.

[2] Russell, S.J. \& Norvig, P., Artificial Intelligence: A Modern Approach, 2nd ed., Prentice Hall: Upper Saddle River, N.J., 2003.

[3] Nakayama, S., Kitamura, R. \& Fujii, S., Drivers' Learning and Network Behavior: A Dynamic Analysis of the Driver-Network System as a Complex System, Transportation Research Record, 1676, pp.30-36, 1999. 
[4] Nakayama, S. and R. Kitamura, A Route Choice Model with Inductive Learning, Transportation Research Record, 1725, pp. 63-70, 2000.

[5] Wahle, J., Bazzan, A., Klugl, F. \& Schreckenberg, M., The Impact of RealTime Information in a Two-Route Scenario Using Agent-Based Simulation, Transportation Research, 10C, pp. 399-417, 2002.

[6] Rossetti, R., Bordini, R., Bazzan, A., Bampi, S., Liu, R. \& Van Vliet, D., Using BDI agents to Improve Driver Modelling in a Commuter Scenario, Transportation Research, 10C, pp. 373-398, 2002.

[7] Cetin, N., Nagel, K., Raney, B. \& Voellmy, A., Large-Scale Multi-Agent Transportation Simulations, Computer Physics Communications, 147, pp. 559-564, 2002.

[8] Wardrop J. G. (1952) Some Theoretical Aspects of Road Traffic Research, Proceedings the Institution of Civil Engineers Part II, pp.325-378. 